\title{
In memoriam Univ.-Prof. Dr. med. Dipl. Chem. Werner A. Kaiser
}

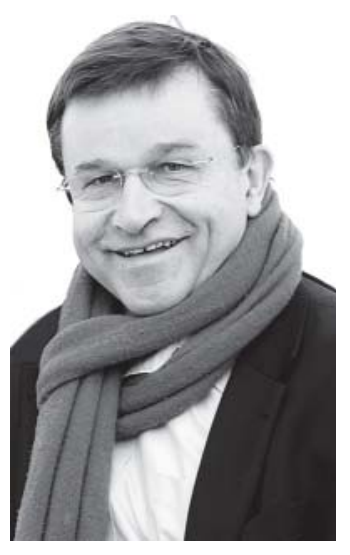

Werner A. Kaiser
Bibliografie

DOI http://dx.doi.org/10.1055/

s-0034-1366222

Fortschr Röntgenstr 2014; 186: 405-406 @ Georg Thieme Verlag KG Stuttgart · New York ISSN 1438-9029

Korrespondenzadresse Univ.-Prof. Dr. med. Dr. med. habil. Dietbert Hahn

Gartenstr.12 97273 Kürnach Tel.: ++49/93 67/1304 Fax: ++49/0211/81/18804 prof.dietbert@hahn-kuernach.de
Nach kurzer, schwerer Krankheit verstarb Werner Kaiser, der Erfinder der MR-Mammografie, am 27.12.2013 in Bonn. 1983 entwickelte er im Forschungslabor von Siemens in Erlangen die MRMammografie und optimierte dieses Verfahren in den vergangenen 30 Jahren. Er hat durch seine zahlreichen wissenschaftlichen Publikationen, Vorträge und Work-shops der MR-Mammografie weltweit zum Durchbruch verholfen. Die MR-Mammografie hat zu einer eindrucksvollen Verbesserung der Früherkennung des Mammakarzinoms geführt. Durch seine Erfindung konnten viele Frauen früher und damit erfolgreicher therapiert werden und in vielen Fällen ihr Leben gerettet werden.

Die Radiologie verliert mit Werner Kaiser einen eindrucksvollen, aber immer bescheiden gebliebenen Menschen, einen charismatischen Arzt und herausragenden Forscher.

Werner Kaiser wurde am 5.10.1949 in Bühl im Badener Land geboren. Er besuchte von19561969 die Volksschule und das Gymnasium in Bühl. Die Schulzeit und einen Teil seines Chemiestudiums verbrachte er in seiner geliebten Heimat. Von 1969 - 1980 studierte er parallel Chemie und Medizin in Karlsruhe und Freiburg. Seine Diplomarbeit in Organischer Chemie an der Universität Freiburg 1975 wurde mit der Note sehr gut bewertet, seine experimentelle Doktorarbeit am Biochemischen Institut der Universität Freiburg 1978 mit der Note summa cum laude.

Nach dem erfolgreichen Abschluss des Medizinstudiums startete Werner Kaiser seine klinische Weiterbildung von 1980 - 1983 in der Allgemeinmedizin, Inneren Medizin und Kinderheilkunde.

Seine Weiterbildung zum Facharzt für Radiologie begann im Klinikum rechts der Isar bei Prof. Dr. Hermann Anacker vom 1.9.1983 - 30.11.1983. Da Werner Kaiser unbedingt wissenschaftlich in der MR-Tomografie arbeiten wollte, wechselte er zum 1.12.1983 an das Klinikum Nürnberg zu Prof. Dr. Eberhard Zeitler. Am 21.9.1988 erhielt er die Anerkennung als Arzt für Radiologische Diagnostik.

Seine Kenntnisse in Medizin und Chemie konnte er in seinem großen Forschungsgebiet der Magnetresonanztomografie und MR-Spektroskopie in idealer Weise verbinden. Parallel zu seiner klinischen Weiterbildung war er seit 1983 wissenschaftlich im Forschungslabor bei Siemens in Erlangen tätig und hat wesentliche Entwicklungsarbeit in der Anfangszeit der MR-Tomografie geleistet. Bereits 1985 veröffentlichte Werner Kaiser die ersten wissenschaftlichen Ergebnisse seiner MR-Mammografiestudien, die in Zusammenarbeit mit der Gynäkologischen Abteilung des Klinikums Nürnberg durchgeführt wurden. Die MR-Mammografie war sein Lebenswerk, in das er seine ganze Schaffenskraft setzte. In mühevoller Detailarbeit hat er immer wieder neue Verbesserungen erarbeitet. Gegen viele Widerstände, auch aus den Reihen der eigenen Fachkollegen, hat er die MR-Mammografie als Routineverfahren in der modernen Brustkrebsdiagnostik etabliert.

Nach Abschluss seiner Weiterbildung wechselte Werner Kaiser im Januar 1990 an die Radiologische Klinik der Universität Bonn zu Prof. Dr. Max Reiser. Hier habilitierte er sich am 6.11.1991 für das Fach Radiologie mit einer Arbeit „Entwicklung und Optimierung der Magnet-Resonanz-Tomographie der Mamma“ über die von ihm entwickelte und optimierte Methode zur Untersuchung der weiblichen Brust.

Am 1.2.1993 erhielt Werner Kaiser den Ruf auf eine C3-Professur am Institut für Röntgendiagnostik der Bayerischen Julius-Maximilians-Universität Würzburg bei Prof. Dr. Dietbert Hahn. Als Leiter der Abteilung für MR-Tomografie, leitender Oberarzt und stellvertretender Direktor des Instituts hat er sich große Verdienste beim Aufbau der MR-Tomografie am Klinikum der Universität Würzburg erworben. Zugleich konnte er sein Steckenpferd, die MR-Mammografie, weiter ausbauen. Er hat in Würzburg mit sehr großem Erfolg ein Zentrum für moderne Brustkrebsdiagnostik aufgebaut, das neben der MR-Mammografie hochkompetent auch Mammografie, Mammasonografie und alle modernen Biopsieverfahren anbieten kann.

Am 1.11.1994 folgte Werner Kaiser dann einem Ruf auf die C4-Professur für Radiologie an der Friedrich-Schiller-Universität Jena. Bis zu seinem Tod hatte er den Lehrstuhl für Bildgebende Diagnostik inne und war Direktor des Instituts für Diagnostische und Interventionelle Radiologie.

Das Universitätsklinikum Jena hatte den letzten Lehrstuhlinhaber für Radiologie aus der DDR-Zeit 1989/90 in den Ruhestand verabschiedet. Der Lehrstuhl wurde dann in einer kurzen, nur wenige Monate andauernden Phase durch einen neuen ärztlichen Direktor geleitet. Bedingt durch die politischen Umstrukturierungen im Rahmen der Wende wurde diese Lehrstuhlberufung durch das Land Thüringen nicht aufrechterhalten und die Radiologie ab Februar 1992 nur noch kommissarisch verwaltet. Der Lehrstuhl blieb bis zur Berufung von Werner Kaiser vakant.

Werner Kaiser hat mit dem ihm eigenen Engagement diese Klinik zu neuem Leben erweckt. Dies wurde ihm jedoch vorwiegend lokal nicht von allen gegönnt. Professor Kaiser pflegte auf verschiedenen klinischen und wissenschaftlichen Ebenen mit vielen deutschen Universitätskliniken eine 
enge Zusammenarbeit. Insbesondere hatte sich in den letzten Jahren eine enge Zusammenarbeit mit der Charité entwickelt, von der der Standort Jena sehr profitierte.

Sein Herzblut floss in die Entwicklung der experimentellen Radiologie und die Weiterentwicklung der MR-Tomografie mit dem Schwerpunkt MR-Mammografie. Als Pionier der MR-Mammografie im wissenschaftlichen und im klinischen Bereich war Professor Kaiser nicht nur unter Fachkollegen höchst geschätzt. Seine internationale Reputation wird durch eine enorme Anzahl an wissenschaftlichen Publikationen, Monografien und Vorträgen sowie Kursen in der MR-Mammografie unterstrichen.

Das Publikationsverzeichnis von Werner Kaiser umfasst insgesamt 457 Originalarbeiten in renommierten nationalen und internationalen Journalen, 964 gedruckte Vorträge und Abstrakts, 401 nicht gedruckte Vorträge sowie eine große Zahl von Buchbeiträgen und eigenen Büchern.

Während seines Ordinariats habilitierte er insgesamt 13 Mitarbeiter und betreute selbst 110 Doktoranden. 13 seiner Mitarbeiter übernahmen Chefarztpositionen. Am Institut entstanden Professuren für die Schwerpunkte der Radiologie (Neuroradiologie und Kinderradiologie) aber auch für die wissenschaftlichen Schwerpunkte Medizinische Physik und Experimentelle Radiologie.

Von 1997 - 2012 organisierte er in Jena alle drei Jahre den international herausragenden „International Congress on MR-Mammography“, auf dem national und international anerkannte Experten aus aller Welt die Möglichkeit hatten, ihre Meinungen auszutauschen und wissenschaftlich zu diskutieren.

Aufgrund seines großen Engagements gelang Prof. Kaiser eine nachhaltige Stärkung des wissenschaftlichen Standorts Jena. Das Institut für Diagnostische und Interventionelle Radiologie am Universitätsklinikum entwickelte sich unter seiner Leitung zum technisch bestausgestatteten radiologischen Institut in den neuen Bundesländern mit zahlreichen Hochleistungsgroßgeräten. Prof. Kaiser hinterlässt dem Universitätsklinikum Jena ein hervorragend ausgestattetes MR-Forschungszentrum, in dem sich ein Teil seines akademischen Wirkens widerspiegelt und in dem er in den letzten Jahren die MR-Mammografie betrieb. Er hat dieser Klinik seine Berufsjahre als ärztlicher Direktor in übergroßem Maße gewidmet. Für diese ungeheure Lebensleistung gebührt ihm die höchste Anerkennung und Wertschätzung.

Die MR-Mammografie blieb aber auch während der letzten beiden Jahre sein wissenschaftlicher Schwerpunkt. Inzwischen hatte sich die Methode klinisch etabliert und Patientinnen aus ganz Deutschland, aber auch aus Nachbarländern, wurden zu ihm nach Jena überwiesen. Hohe Untersuchungszahlen und ein Team von exzellenten Schülern waren der Nährboden für eine Reihe von klinisch wichtigen Studien zur MR-Mammografie in den letzten Jahren.

Werner Kaiser betreute den Großteil seiner Patientinnen selber, indem er in den sogenannten „Mamma-Wochen“ ganze Tage an den MR-Geräten verbrachte und in einem höchst standardisierten Modus bis zu 50 MR-Mammografien am Tag durchführte.
Die wissenschaftlichen Auswertungen erfolgten dann in nächtlichen Sitzungen zusammen mit seinen Schülern.

Ein Credo von Werner Kaiser war, dass die MR-Mammografie der Röntgenmammografie in der Früherkennung von Karzinomen weit überlegen sei. Seine Schüler wissen, dass er nie daran gezweifelt hat, dass die MR-Mammografie einmal die Methode der Wahl für die Brustkrebsdiagnostik aller Frauen werden würde. Ein zweites Credo von Werner Kaiser war, dass für die Differenzierung von Mammaläsionen in der MR-Tomografie nicht nur die Kontrastmittelkinetik betrachtet werden darf, sondern morphologische Merkmale hinzugezogen werden müssen. Mithilfe des von ihm erstellten Klassifikationsschemas kann eine hohe Genauigkeit in der Differenzierung von Malignität und Benignität erreicht werden.

Bis zuletzt liebte er die argumentative Auseinandersetzung über Sensitivität und Spezifität der MR-Mammografie. Die Beschränkung der Beurteilung von Läsionen auf die Beurteilung der Kontrastmittelkinetik ist denn auch eine Erklärung für die überholte Meinung, dass die MR-Mammografie zwar eine hohe Sensitivität, aber eine niedrige Spezifität aufweise. Vergleicht man die Befundgenauigkeit von unerfahrenen mit sehr erfahrenen Befundern, so zeigt sich, dass auch der Unerfahrene eine hohe Sensitivität erreichen kann. Aber nur der Erfahrene kann mithilfe morphologischer Kriterien auch eine hohe Spezifität von über 95\% erreichen. Neben vielen anderen Ehrungen und Anerkennungen erhielt Werner Kaiser 1991 den European Magnetic Resonance Award für die Entwicklung der MR-Mammografie, 2003 den Eugenie und Felix Wachsmann Preis der Deutschen Röntgengesellschaft und im selben Jahr die Atis K. Freimanis Gastprofessur an der Michigan State University. 2006, 2010 und 2011 war er Visiting Professor an der Harvard Medical School in Boston.

In Fortsetzung hoher Ehrungen und Auszeichnungen durch verschiedene Fachgesellschaften während seiner Tätigkeit und in Anerkennung seiner besonderen Verdienste auf dem Gebiet der MRMammografie wurde Professor Kaiser posthum am 28.1.2014 das Bundesverdienstkreuz am Bande verliehen.

Trotz aller Belastungen ruhte Werner Kaiser vor allem in seiner Familie. Er schwärmte immer von seiner lieben Ehefrau, seinen fünf Kindern und den vier Enkelkindern, um die er sich bis zum Schluss rührend kümmerte.

Wir werden uns stets an seinen beharrlichen, visionären Blick für sein Fachgebiet und seinen Forscherdrang erinnern, genauso wie an sein umfassendes Fachwissen, das er stets überzeugend und passioniert vermitteln konnte. Die akademische Welt verliert mit Professor Werner A. Kaiser einen engagierten Hochschullehrer und renommierten Wissenschaftler. Seine Patientinnen und Patienten werden ihn als vertrauensvollen und einfühlsamen Arzt vermissen. In ehrender Erinnerung verneigen wir uns vor seinem Lebenswerk.

D. Hahn, B. Hamm, C. Kaiser, M. Langer, D. Maintz, U. Teichgräber 